\title{
Experimental and Theoretical Study of the Yellowing of Ancient Paper*
}

\author{
A. Mosca Conte, O. Pulci, R. Del Sole \\ ETSF, Dipartimento di Fisica, Università di Roma Tor Vergata, \\ Via della Ricerca Scientifica 1, I-00133 Rome, Italy \\ A. Knapik, J. Bagniuk, J. Lojewska \\ Faculty of Chemistry, Jagiellonian University, Ingardena 3, 30-060 Kraków, Poland
}

\author{
L. Teodonio \\ Istituto centrale per il restauro e la conservazione del patrimonio archivistico e librario (IC-RCPAL), \\ Italian Minister for Cultural Heritage, Via Milano 76, 00184 Rome, Italy
}

\section{Missori ${ }^{\dagger}$}

Istituto dei Sistemi Complessi, Consiglio Nazionale delle Ricerche, Via Salaria Km 29.300, 00016 Monterotondo Scalo (Rome), Italy

(Received 16 July 2012; Accepted 21 September 2012; Published 13 October 2012)

\begin{abstract}
Paper is the most widely used writing support due to the remarkable properties of its principal component cellulose - one of the most abundant biomaterials present on Earth. However, due to the complexity of the material, an exhaustive picture of its degradation pathways is still missing. In this paper, we will present recent results and progresses obtained in the comprehension of the role of cellulose oxidation in the yellowing of ancient paper. Visible and ultraviolet spectra of cellulose in ancient paper samples and reference modern samples artificially aged have been interpreted with the aid of ab-initio Time-Dependent Density Functional Theory calculations. Through the comparison of measured and calculated absorption spectra, several oxidized forms of cellulose polymers, acting as chromophores, and responsible for ancient paper yellowing were identified. The relative concentration of ketones and aldehydic groups depends on the environmental conditions in which samples were stored along their life.
\end{abstract}

[DOI: $10.1380 /$ ejssnt.2012.569]

Keywords: Density functional calculations; Reflection spectroscopy; Biological compounds; Porous solids; Oxidation; Aldehydes; Ketones; Carboxylic acid

\section{INTRODUCTION}

Protecting ancient paper artefacts from decay poses a serious problem for libraries, archives and museums all over the world. In fact, paper has been the most widely used writing support since the first centuries A.D. in China, afterwards in Japan at the beginning of $7^{\text {th }}$ century, and since the Middle Ages in the Western World, with the result that a huge number of books, documents and drawings have been accumulated along the centuries $[1,2]$. The preservation of these cultural properties poses the significant challenge of limiting the deterioration of the paper materials. This task must be based on an advanced knowledge of the microscopical characteristics of paper materials and their degradation processes.

Paper is a complex multi-component material consisting in a felt of cellulose fibers derived from plants in ancient times and wood pulp today. Depending on the production period and technology employed, the paper composition varies. The ancient hemp, cotton or linen based papers contain mainly cellulose ( $>90 \%$ in weight) with the addition of animal glue and alum as sizing compounds, while modern soft or hardwood ones may also include lignin $(<30 \%$ in weight $)$ and other additives [3].

Cellulose is a biopolymer present in the primary cell

*This paper was presented at the 16th International Conference on Solid Films and Surfaces (ICSFS-16), University of Genoa, Genoa, Italy, 1-6 July, 2012.

${ }^{\dagger}$ Corresponding author: mauro.missori@isc.cnr.it

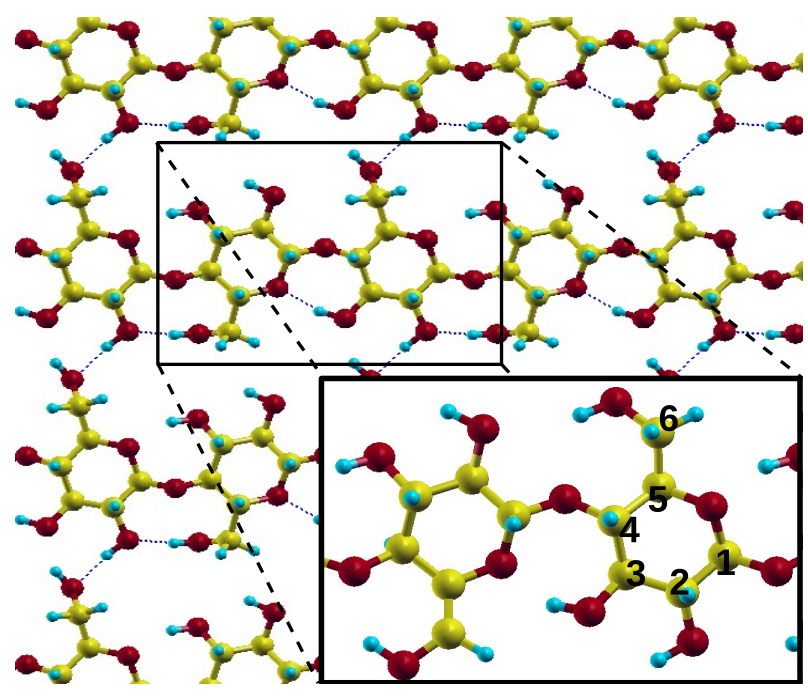

FIG. 1: Structure of cellulose crystal. Yellow, red, and cyan balls correspond respectively to $\mathrm{C}, \mathrm{O}$, and $\mathrm{H}$ atoms. Blue dashed lines indicate $\mathrm{H}$-bonds. The $\mathrm{C}$ atoms numbering is indicated in the inset.

wall of green plants. It is made of several hundred to over thousands units of $\beta$-(1,4)-D-glucopyranose (Fig. 1). Cellulose is the most common organic compound on Earth, representing $40 \%$ of the annual production of biomass [4].

Inter-chain secondary valence hydrogen bonds between cellulose polymers is the principal mechanism responsible for cellulose's supramolecular arrangements in microfibrils, which in turn are composed of an assembly of 
highly ordered (crystalline) domains, and of disordered (amorphous-like) regions. In the case of flax and cotton, the crystalline domains have dimensions of $2-4 \mathrm{~nm}$ in cross section and $100 \mathrm{~nm}$ in length. Microfibrils are bonded up to form fibers whose diameter ranges from $\sim 1$ to $\sim 10 \mu \mathrm{m}$ $[5]$.

Paper degradation occurs by the weakening of mechanical properties of the sheets and by yellowing. The overall process of paper degradation may be seen as a combination of acid hydrolysis, which shortens the cellulose polymeric chains weakening the mechanical properties of the sheets, and oxidation with subsequent development of chromophores and other byproducts, and discoloration of the remaining substratum. These two degradation routes are interdependent as among the oxidation by-products are carboxylic acids and autoxidation species, which in turn promote acid catalysed hydrolysis and autoxidation, respectively.

The mechanism of acidic hydrolysis is rather known $[6,7]$. Instead, cellulose oxidation running through the radical mechanism initiated by active oxygen species is a complex process with many possible reactions still to be clarified [7-9].

The moderate oxidation of cellulose causes the formation of a broad ultraviolet (UV) absorption band with a peak around $4.8 \mathrm{eV}(260 \mathrm{~nm})$, and a long absorption tail towards lower energies up to the visible region. The absorption peak at $4.8 \mathrm{eV}$ has been attributed to the development of carbonyl groups through the comparison of the spectra of similar compounds [10]. On the other hand, pure cellulose shows no absorption bands in the ultraviolet-visible (UV-Vis) region up to around $6 \mathrm{eV}$ (200 nm) [7]. Vibrational spectroscopy and chemical analysis have been carried out, in an attempt to clarify the configuration within the glucopyranose ring of the carbonyls responsible for the observed absorption spectrum in moderate oxidation. [8, 11-13].

However, because of the important role of the local chemical environment around the oxidized groups, this procedure prevents a full band assignment and does not allow to distinguish, for example, between ketones and other enolic groups in the oxidized cellulose. A better knowledge of the oxidation products of cellulose in ageing could shed light to the mechanisms in play during paper degradation, useful for the improvement of conservation and restoration practice.

In this paper, we will present recent results and progresses obtained in the comprehension of the role of cellulose oxidation in the yellowing of ancient paper. To this goal, UV-Vis spectra of degraded cellulose in modern and ancient samples have been measured, and interpreted with the aid of Time-Dependent Density Functional Theory (TDDFT) calculations [14]. We will show that, by using a combined experimental and theoretical approach, important information on the conservation history of ancient paper samples can be recovered.

\section{EXPERIMENTAL}

The ancient specimens used in this research consist of dated and identified paper, produced during the $15^{\text {th }}$ century in European countries, showing various levels of degradation and discoloration. All the samples used in the experiments are listed in Table I. The ancient paper used in the experiments carried no printing.

Due to the fact that the environmental conditions to which the ancient samples were subjected throughout their history are unknown, an artificial high-temperature degradation process was performed on modern paper samples to allow for a constant monitoring of environmental conditions during aging and to differentiate the role of the different aging factors in the resulting degradation [7].

Modern paper samples, obtained from Netherlands Organization for Applied Scientific Research (TNO), are made of unbleached cotton linters, containing very low inorganic ingredients (ash content $<0.005 \%$ in weight) and no additives or lignin. These specimens, which are made of cellulose very similar to that used in the past times, are named, following [15], P2. These samples were artificially aged up to 48 days in three different reactors with different environmental conditions as reported in Table II.

Different ageing condition were used to be able, at least partly, to discriminate among different degradation routes. At dry air atmosphere we expect development of carbonyl groups, while at humid atmosphere $(\mathrm{RH}=59 \%)$ high contribution of hydrolysis of glycosidic bonds is expected. The temperature and relative humidity were chosen according to our previous studies $[11,12]$. The open reaction chamber was used to eliminate the influence of gaseous products of paper degradation on the degradation progress, in contrast to closed vessel in which volatiles, especially acidic ones, additionally degrade paper samples. Thus in closed vessel purged with air and moisture the highest degradation rate is expected. Additionally, aging conditions were designed as a compromise in order to get measurable changes in the UV-Vis spectra of model paper samples within a reasonable period of time. At the chosen temperature of $90^{\circ}$ a moderate oxidation of the cellulose functional groups towards the carbonylic groups of various degrees of freedom and chemical environment is expected $[12,16]$.

The absorbance $\left(A_{K M}\right)$ of modern and ancient paper samples was obtained by applying the Kubelka-Munk (KM) theory to diffuse reflectance measurements [17, 18]. Ultraviolet-visible-near infrared (UV-Vis-NIR) diffuse reflectance spectra were measured at room temperature and at $50 \%$ of relative humidity $(\mathrm{RH})$ conditions by using a Jasco V-570 spectrophotometer equipped with an integrating sphere and a $\mathrm{PbS}$ detector or by using an Avantes set-up formed by an AvaLight-DH-S-BAL light source, an AvaSphere-30-REFL integrating shere and a AvaSpec2048x14-USB2 spectrometer.

Two sets of spectra were measured, one with the paper samples placed over a background with reflectance close to 1 (white), and another with the paper samples placed over a background with reflectance close to 0 (black). The backgrounds reflectance were measured separately. All measured spectra were normalized to a Spectralon diffuse reflectance reference standard (factory calibrated). The spectra resolution was set to $2 \mathrm{~nm}$. The KM model was used to convert the reflectance of a sample over the two backgrounds into the reflectance that would have an infinite layer of same sample $\left(R_{\text {inf }}\right)$ to recover the KM 
TABLE I: List of all the ancient samples analyzed.

\begin{tabular}{ccc}
\hline \hline Sample label & Origin & Date \\
\hline A1 & Perpignan (France) & 1413 \\
A2 & Milan (Italy) & 1430 \\
A3 & Milan (Italy) & 1430 \\
B1 & Pula (Croatia) & $1441-1446$ \\
B2 & Gaeta (Italy) & 1480 \\
\hline \hline
\end{tabular}

TABLE II: List of the modern samples (P2) analyzed. Environmental conditions used in artificially aging the samples are listed. $\mathrm{RH}$ indicates the relative humidity

\begin{tabular}{clll}
\hline \hline $\begin{array}{c}\text { Aging process } \\
\text { label }\end{array}$ & Reactor Type & $\begin{array}{l}\text { Environmental } \\
\text { conditions }\end{array}$ & $\begin{array}{l}\text { Expected degradation } \\
\text { factors }\end{array}$ \\
\hline $\mathrm{D}$ & Open drier & Dry air, $\mathrm{T}=90^{\circ} \mathrm{C}$ & Air \\
$\mathrm{C}$ & Open climatic chamber & Air, $\mathrm{RH}=59 \%, \mathrm{~T}=90^{\circ} \mathrm{C}$ & Air and humidity \\
$\mathrm{V}$ & Closed vial & Air, $\mathrm{RH}=59 \%, \mathrm{~T}=90^{\circ} \mathrm{C}$ & Air, humidity and gaseous byproducts \\
\hline \hline
\end{tabular}

absorbance spectra $A_{K M}$

$$
A_{K M}=\frac{\left(1-R_{\mathrm{inf}}\right)^{2}}{2 R_{\mathrm{inf}}}
$$

which can be finally compared with numerical simulations [18].

\section{THEORETICAL METHOD}

We used ab-initio (free-parameter) theoretical computational techniques based on Density Functional Theory (DFT) [19] and on Time-Dependent DFT (TDDFT) [14] to calculate respectively ground-state and optical properties. We have calculated the macroscopic dielectric function of cellulose by using the Casida algorithm [20] within a TDDFT framework with a gradient-corrected local approximation (BLYP) [21] for the exchange-correlation potential. The theoretical absorbance $\alpha^{\text {theor }}$ has then be obtained by the expression:

$$
\alpha^{\text {theor }}=\frac{4 \pi}{\lambda} \sqrt{\frac{\sqrt{\varepsilon_{1}^{2}+\varepsilon_{2}^{2}}-\varepsilon_{1}}{2}},
$$

where $c$ is the speed of light, $\lambda$ is the wavelength of external radiation, $\varepsilon_{1}$ and $\varepsilon_{2}$ are respectively the real and the imaginary part of the complex dielectric constant.

We have considered several possible oxidized groups of cellulose which are supposed to give absorption effects in the visible range $[8,12]$. These are shown in Fig. 2 and are named as reported in Table III.

As a structural model for the simulation of optical properties we have chosen an infinite crystal of cellulose as an approximation of cellulose crystalline domains. Cell parameters of the cellulose crystal were taken from X-ray diffraction data [4] and refer to the monoclinic crystallographic phase called cellulose- $\mathrm{I}_{\beta}[4,5]$.

The atomic coordinates inside the cell, containing 42 atoms, were theoretically calculated by the QUANTUM ESPRESSO plane-wave DFT code [22].
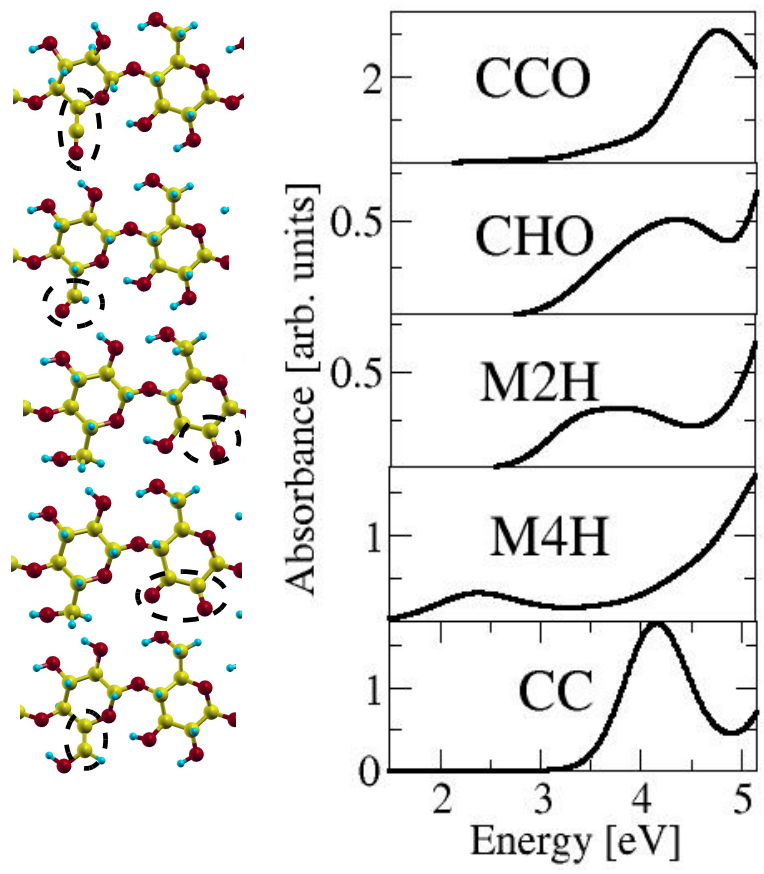

FIG. 2: Theoretical absorbance spectra of oxidized groups shown in the left side.

Further theoretical details on the method used are reported in $[15,23]$.

\section{RESULTS AND DISCUSSION}

In Fig. 3(a) we report $A_{K M}$ for all ancient samples. The absorbance has a maximum value at the high energy side of the measured spectra. At lower energies it smoothly decreases with a tail extending up to the begin- 
TABLE III: Description of the oxidized groups of cellulose considered in the simulations (Fig. 2).

\begin{tabular}{cc}
\hline \hline Oxidized group & Description \\
\hline $\mathrm{CCO}$ & complete oxidation of $\mathrm{C}(6)$ with carbonylic group and a double bond $\mathrm{C}(5)=\mathrm{C}(6)(\mathrm{C}(5)=\mathrm{C}(6)=\mathrm{O})$ \\
$\mathrm{CHO}$ & oxidation of $\mathrm{C}(6)$ with the formation of an aldehydic group $(\mathrm{C}(6)=\mathrm{O})$ \\
$\mathrm{M} 2 \mathrm{H}$ & oxidation to a single ketone on $\mathrm{C}(2)(\mathrm{C}(2)=\mathrm{O})$ \\
$\mathrm{M} 4 \mathrm{H}$ & conjugated diketones on $\mathrm{C}(2)$ and $\mathrm{C}(3)(\mathrm{O}=\mathrm{C}(2)-\mathrm{C}(3)=\mathrm{O})$ \\
$\mathrm{CC}$ & formation of a double bond $\mathrm{C}(5)=\mathrm{C}(6)$ \\
\hline \hline
\end{tabular}

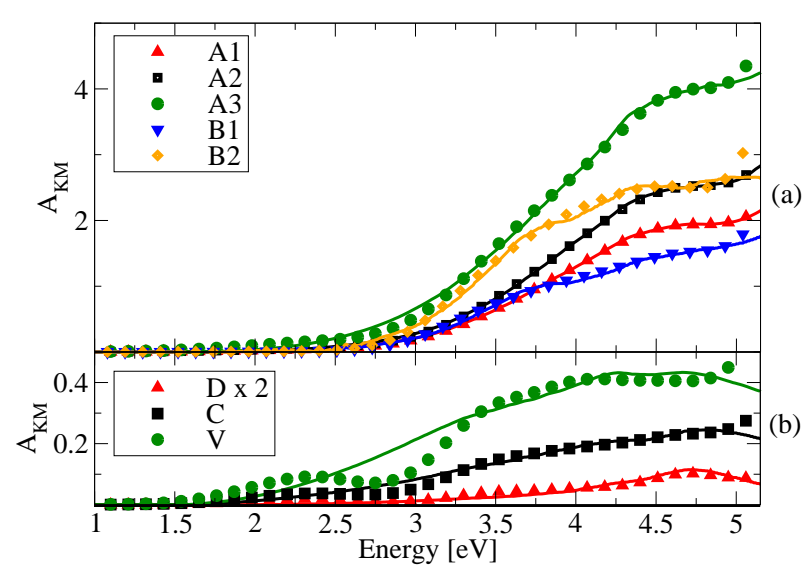

FIG. 3: (a) Ancient samples: experimental absorbance $A_{K M}$ (solid line) and corresponding theoretical absorption spectra (symbols). (b) Modern samples: experimental absorbance $A_{K M}$ (solid line) and corresponding theoretical absorption spectra (symbols).

ning of the visible energy region between 2.5 and $3 \mathrm{eV}$.

In Fig. 3(b) spectra for samples artificially aged for 48 days in $\mathrm{D}, \mathrm{C}$ and $\mathrm{V}$ conditions (Table II) are reported. Spectra for unaged samples are not reported because they do not show any absorption below $5 \mathrm{eV}$. All absorbance spectra of modern samples artificially aged consist of a broad peak around $4.8 \mathrm{eV}(260 \mathrm{~nm})$ already attributed to the development of carbonyl groups in glucopyranose units [10]. For samples aged in $\mathrm{C}$ and $\mathrm{V}$ conditions an additional peak at $4.2 \mathrm{eV}(295 \mathrm{~nm})$ appears. Moreover, a smooth and long tail reaching the visible energies below $2 \mathrm{eV}(620 \mathrm{~nm})$ is present for the $\mathrm{V}$ sample. The wide energy range of the spectral region of absorbance suggests the presence of several chromophores of different nature.

The calculated optical absorption spectra do not present absorption below $5.1 \mathrm{eV}$ for unaged cellulose, in agreement with the experiments. The oxidized groups, instead, are characterized by the appearance of maxima between 2 and $5 \mathrm{eV}$ as shown in Fig. 2. The hole-electron charge distribution related to these peaks is mainly localized on the double bonds induced by the oxidation. Because of their strong localization, the optical absorption spectrum below $5 \mathrm{eV}$ of every oxidized group can be considered as produced by several isolated not-interacting chromophores [8, 12, 15].

Below this energy threshold the absorption bands from different oxidized groups are clearly distinguishable over a null background. Below $5.15 \mathrm{eV}$, the optical transi- tions involve only electronic states related to the oxidized groups, which are responsible for the yellowing of paper. As a consequence of the fact that the optical bands below $5.15 \mathrm{eV}$ are a kind of "fingerprint" of the oxidized groups studied, whose lower energy electronic states are very localized, a minimal square algorithm could be applied to extrapolate their relative concentrations by a comparison with the optical experimental spectra, as better explained below. Above $5.15 \mathrm{eV}$, instead, bands due to oxidized groups are superimposed with the fundamental absorption of the host material (cellulose).

The lack of interactions between the chromophore groups inferred from the simulations allows us to consider the optical absorption spectrum of aged cellulose as a linear combination of the spectra calculated for every single oxidized group in the cellulose backbone (Fig. 2). This observation enabled us to compute the theoretical optical absorption spectra by minimizing the sum of squared residuals $S$ :

$$
S=\sum_{j}\left(A_{K M}^{\exp }\left(\lambda_{j}\right)-\sum_{i} b_{i} \alpha_{i}^{\text {theor }}\left(\lambda_{j}\right)\right)^{2},
$$

where $A_{K M}^{e x p}$ is the experimental value obtained by the Kubelka-Munk model (Eq. 1) for each value of the wavelength $\lambda_{j}$, where $j$ is the discretization index of the wavelength, $\alpha_{i}^{\text {theor }}$ are the theoretical absorbances obtained by solving Eq. 2 for each oxidized group, and $b_{i}$ are the linear combination coefficients to be found by minimizing the above expression (index $i$ runs over the five oxidized groups). By normalizing the linear combination coefficients $b_{i}$, the relative concentrations of chromophores producing yellowing on the samples after aging can be estimated.

In Fig. 3(a), and (b), the symbols represent the relative concentrations of chromophores that better reproduce the experimental spectrum obtained by minimizing Eq. 3 in the energy range 1.5-5.1 eV. A very good agreement between calculated and experimental spectra is found for all samples.

In particular, our calculations are able to identify the most significant structure observed in the experimental spectra. M4H was predicted to be the most significant oxidation causing paper yellowing $[8,11,12]$ and to be the only one active in the UV-Vis wavelength range. Instead, we show that other oxidized groups in cellulose can be optically active. The aldehydic CHO group is expected to be the transient product of the subsequent oxidation of the $\mathrm{C}(6)$ to carboxylic group $(\mathrm{COOH})$ in the moderate cellulose oxidation [12]. Interestingly, simulations performed with $\mathrm{COOH}$ showed, instead, no absorption bands 


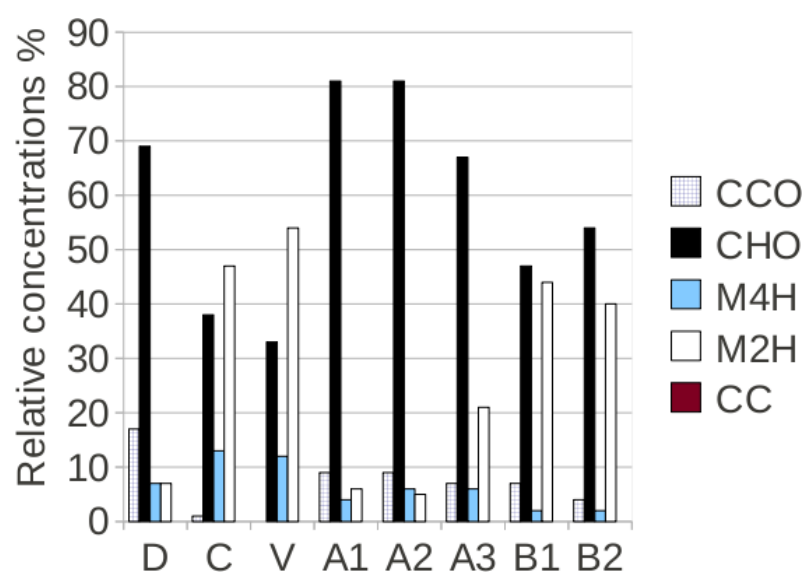

FIG. 4: The relative concentration of the 5 oxidized groups acting as chromophores as calculated from simulations for modern samples and ancient samples.

in the UV-Vis range up to $5.1 \mathrm{eV}$ and therefore no optical contribution to paper yellowing.

For sample V, we observe a small difference between experimental and theoretical absorption spectra in the range 2.5-3 eV. We attribute this mismatch to the presence of additional degradation byproducts, trapped in the sample because of the closed environment aging condition. The volatile byproducts detected by gas chromatographymass spectrometry (GC-MS) measurements during ageing tests performed in closed vials (Table II) include aliphatic hydrocarbons, alcohols, aldehydes, ketones, carboxylic acids, esters, terpens and aromatic compounds from several to over a doyen carbon atoms in a molecule [24]. These to a high extent leave the environment of the sample. Other heavier products of simultaneous oxidation and glucopyranose breakage include oligomers are also supposed to be formed in paper [25] but their contribution to the overall oxidation is rather low [26]. We will address this topic in future works.

In Fig. 4 we report the relative concentrations of chromophores, obtained by normalizing the linear combination coefficients of theoretical spectra. Very different results appear when comparing modern samples aged in various environmental conditions. The $\mathrm{CHO}$ groups emerge as the most abundant species in the sample aged under pure oxidative conditions (D). Humidity present in $\mathrm{C}$ and $\mathrm{V}$ environments appears to favor the oxidation towards of $\mathrm{M} 2 \mathrm{H}$ and also, to a less extent, $\mathrm{M} 4 \mathrm{H}$ chromophores, inhibiting the $\mathrm{CHO}$ and causing almost complete disappearance of CCO groups. Apparently, water molecules provide additional oxygen radicals which facilitate the formation of ketones on glucopyranose rings [27]. Indeed, water can also be regarded as the transporting agent for both protons and active oxygen for oxidation [12].

In ancient samples $\mathrm{A} 1, \mathrm{~A} 2$ and $\mathrm{A} 3, \mathrm{CHO}$ has been found to be the most abundant oxidized group. Coming from A1 to A3 sample, the increasing yellowing can be accounted for by the increasing contribution of ketones $\mathrm{M} 2 \mathrm{H}$.

This increase is even larger in B1 and B2 ancient samples, where the relative concentrations of $\mathrm{M} 2 \mathrm{H}$ and $\mathrm{CHO}$ appear to be very similar to those of modern samples aged in humid conditions (see Fig. 4). We also note that the CC oxidized group is always absent in all samples, regardless of their origin.

While in the "A" ancient samples the concentration of $\mathrm{M} 2 \mathrm{H}$ is very low compared to $\mathrm{CHO}$, in "B" samples these two concentrations are comparable. Historical reports on the conservation of these sample suggest that the first have been conserved in dry conditions, while the second in a more humid atmosphere. This suggestion is reinforced by the comparison with modern samples artificially aged. In any case, the above observations show that the study of relative concentrations of chromophores can be used as an indicator of the conservation history of a manuscript.

\section{CONCLUSION}

We related optical reflectivity experimental spectra of aged paper to the chemical transformation that caused optical visual changes on it by a comparison with theoretical simulations based on TDDFT.

We identified the oxidized groups mainly responsible for visual damages in aged paper and observed that the formation of some of these groups is favored by some particular conservation conditions. We therefore set up a diagnostic procedure also allowing to get hints on the historical conditions of ancient paper documents that is totally non-invasive and non-destructive, and is therefore suitable to determine the state of conservation of ancient fragile and unique manuscripts.

In addition, this knowledge could be used for the generation of a new class of bleaching and reducing agents to be employed in paper conservation and restoration techniques.

\section{Acknowledgments}

We acknowledge CPU time granted by CINECA, and funding from the EC's FP7 grants n. 211956 (ETSF [29] user project 211) and from the IC-RCPAL (Italian Minister for Cultural Heritage).
[1] D. Hunter, Papermaking: The History and Technique of an Ancient Craft (Dover Publications, New York, 1978).

[2] S. Yagihashi, Handbook of the Art of Washi (All Handmade Washi Association, Tokyo, 1991), p. 72.

[3] G. Righini, A. L. Segre, G. Mattogno, C. Federici, and P. F. Munafó, Naturwissenschaften 85, 171 (1998).

[4] H. A. Krassig, Cellulose (CRC Press, 1993).
[5] A. C. O'Sullivan, Cellulose 4, 173 (1997).

[6] Y.-Z. Lai, in: Wood and Cellulosic Chemistry, Eds. D. N.-S. Hon and N. Shiraishi (Dekker, New York, 2000).

[7] S. Zervos, in: Cellulose: Structure and Properties, Derivatives and Industrial Uses, Eds. A. Lejeune and T. Deprez (Nova Publishing, New York, 2010), p. 155.

[8] T. Lojewski, K. Zieba, A. Knapik, J. Bagniuk, A. Luban- 
ska, and J. Lojewska, Appl. Phys. A 100, 809 (2010).

[9] M. Strlic and J. Kolar (Eds.), Aging and Stabilisation of Paper (National and University Library, Ljubljana, 2005)

[10] A. Bos, J. Appl. Pol. Sci. 16, 2567 (1972).

[11] J. Lojewska, M. Missori, A. Lubanska, P. Grimaldi, K. Ziba, L. M. Proniewicz, and A. Congiu Castellano, Appl. Phys. A 89, 883 (2007).

[12] T. Lojewski, P. Miskowiec, M. Missori, A. Lubanska, L. M. Proniewicz, and J. Lojewska, Carbohydrate Polymers 82, 370 (2010).

[13] S. Margutti, G. Conio, P. Calvini, and E. Pedemonte, Restaurator 22, 67 (2001).

[14] E. Runge and E. K. U. Gross, Phys. Rev. Lett. 52, 997 (1984).

[15] A. Mosca Conte, O. Pulci, A. Knapik, J. Bagniuk, R. Del Sole, J. Lojewska, and M. Missori, Phys. Rev. Lett. 108, 158301 (2012).

[16] J. Lojewska, P. Miskowiec, T. Lojewski, and L. M. Proniewicz, Polymer Degradation and Stability 88, 512 (2005).

[17] G. Kortum, Reflectance Spectroscopy (Principles, Methods, Applications), (Springer-Verlag, Berlin-HeidelbergNew York, 1969), p. 111.

[18] B. Philips-Invernizzi, D. Dupont, and C. Cazé, Opt. Eng. 40, 1082 (2001).

[19] P. Hohenberg and W. Kohn, Phys. Rev. 136, B864 (1964); W. Kohn and L. J. Sham, Phys. Rev. 140, A1133 (1965).

[20] M. E. Casida, in: Recent Developments and Applications of Modern Density Functional Theory, Ed. J. M. Seminario (Elsevier, 1996).
[21] C. Lee, W. Yang, and R. G. Parr., Phys. Rev. B 37, 785 (1988).

[22] QUANTUM ESPRESSO: http://www.quantumespresso.org

[23] For DFT calculations we used the plane-wave DFT code espresso [22] with norm-conserving pseudopotentials and an energy cutoff over the wavefunctions of $40 \mathrm{Ry}$, corresponding to 13144 plane waves for the wavefunctions. TDDFT calculations have been performed by the dpforexc code [28] with 4000 plane waves for the exchangecorrelation kernel, and by using 15 occupied and 15 unoccupied bands in the Casida algorithm. For both DFT and TDDFT calculations we used $14 \mathrm{k}$-points in the irreducible Brillouin zone.

[24] T. Lojewski, T. Sawoszczuk, J. M. Lagan, K. Zieba, A. Baranski, and J. Lojewska, Appl. Phys. A 100, 873 (2010).

[25] P. Miskowiec, MSc Thesis (Faculty of Chemistry, Jagiellonian University, Krakow, Poland, 2003).

[26] T. Rosenau, A. Potthast, K. Krainz, Y. Yoneda, T. Dietz, Z. Peralta-Inga Shields, and A. D. French, Cellulose 18, 1623 (2011).

[27] D. Kočar, M. Strlič, J. Kolar, J. Rychlý, L. MatisováRychlá, and B. Pihlar, Polym. Degrad. Stab. 88, 407 (2005).

[28] DP: http://dp-code.org

[29] A. Y. Matsuura, N. Thrupp, G. Xavier, Y. Pouillon, G. Bruant, and G. Onida, Comput. Sci. Eng. 14, 22 (2012). 